\title{
A viscoelastic constitutive model with nonlinear evolutionary internal variables
}

\author{
P. J. Wei, Beijing, and J. K. Chen, Yangzhov, China \\ Received July 24, 2002; revised December 18, 2002 \\ Published online: August 18, 2003 (C) Springer-Verlag 2003
}

\begin{abstract}
Summary. Based on the internal variable theory, a viscoelastic constitutive model of a highly deformable continuous medium is proposed. A set of second rank tensorial internal state variables corresponding to Biot's strain is introduced, and a nonlinear evolution law for these internal variables is suggested. The proposed model may be considered as an extension of the network theory of rubber elasticity to take the viscous effects into account. In order to verify the validity of the present model, uniaxial tension tests for HDPE are carried out at different strain rates. The prediction of the present model shows a good agreement with the experimental data. Finally, a discussion of the present constitutive model is given. It is found that the present constitutive model is more flexible to describe the strain rate sensitivity of polymeric materials in a wide range of strain rates.
\end{abstract}

\section{Introduction}

The nonlinear viscoelastic constitutive relations are often used to describe the mechanical behavior of polymeric materials. Several viscoelastic constitutive theories have been proposed in the past years [1]-[8]. Among these theories, the internal variable theory, which is based on thermodynamic framework, may be considered to be a widely accepted one [5]-[8]. Holzapfel and Simo [7] proposed a fully coupled thermo-mechanical constitutive model which applied to highly deformable bodies with viscous dissipation. The Helmholtz free energy in their constitutive model depends not only on temperature and strain, but also on internal state variables. Nevertheless, Huang et al. [8] pointed out that if a quadratic expression in terms of internal variables in the expression of the free energy is used, the corresponding constitutive relation obtained can only be interpreted as a combination of linear spring and linear dashpot, so the nonlinear elastic effect on the evolution of internal variables cannot be included. In order to overcome the shortcomings, a new expression of the free energy motivated by the molecular network analysis for solid polymers is constructed in [8] to take into account the nonlinear elastic behavior. However, the evolution laws of the internal variable in their constitutive models are assumed to obey the Onsager reciprocal relation which is not enough flexible to describe properly the mechanical behavior of polymeric materials at a wide range of strain rates. In the present paper, a threedimensional constitutive model, which can describe the viscoelastic response of a highly deformable continuous medium at different strain rates, is suggested. A set of second rank tensorial internal state variables corresponding to Biot's strain (engineering strain) is introduced to extend the network theory of rubber elasticity to incorporate the viscous effects. 
A nonlinear evolution equation for these internal variables is proposed instead of the Onsager reciprocal relation and makes the present model more flexible to describe the strain rate sensitivity. In order to confirm the validity of the proposed model, Uniaxial tension tests for HDPE are carried out at different strain rates. The prediction of the present model shows a good agreement with the experimental data. Finally, The effects of various model parameters on describing strain rate sensitivity of materials are discussed. It is found that the present model is more flexible to describe the strain rate sensitivity of polymeric material in a wide range of strain rates.

\section{Formulation of the present viscoelastic constitutive model}

Solid polymers consist of long chains of macromolecules that are connected at junctions provided by the chemical cross-links between macromolecules. A vector from one end of a chain to the other is called the end-to-end vector of a chain. In light of entropy elasticity of rubber material, the deformation of the end-to-end vector induces the change of configuration of network and therefore contributes to the change of entropy significantly. In order to generalize the network theory to take the viscous effect into account, a set of second rank tensorial internal state variables corresponding to Biot's strain is introduced to describe the viscous behavior of these chains. The assumption of affine deformation adopted in the network theory of rubber elasticity is reserved.

Suppose that there are $M$ kinds of chains in the macromolecular network, and the viscous dissipation of the $\alpha$-th kind of chains can be described by a second rank symmetric tensor $\xi_{\alpha}$ called the $\alpha$-th internal variable. The stretch of an individual chain, $\lambda$, directed along a unit vector $\boldsymbol{l}_{\mathbf{0}}$ corresponding to the undeformed configuration, and the $\alpha$-th effective stretch, $\hat{\lambda}_{\alpha}$, may be given by

$\lambda=\left(\boldsymbol{l}_{\mathbf{0}} \cdot \Lambda^{2} \cdot \boldsymbol{l}_{\mathbf{0}}\right)^{\frac{1}{2}}$

$\hat{\lambda}_{\alpha}=\left(\boldsymbol{l}_{\mathbf{0}} \cdot\left(\Lambda-\boldsymbol{\xi}_{\alpha}\right)^{2} \cdot \boldsymbol{l}_{\mathbf{0}}\right)^{\frac{1}{2}}$,

where $\Lambda$ and $\Lambda-\xi_{\alpha}$ are the right stretch tensor and the $\alpha$-th effective right stretch tensor, respectively. Equation (2) implies that the viscous dissipation of the $\alpha$-th kind of chains may be described by the well-known Maxwell model with a spring in series with a dashpot. If the strain energy of a single chain is denoted by $w_{\alpha}^{v}\left(\hat{\lambda}_{\alpha}\right)$, which satisfies $w_{\alpha}^{v}(1)=0$, then the total strain energy of the $\alpha$-th kind of chains may be given by

$W_{\alpha}^{v}\left(\bar{\lambda}_{\alpha}\right)=N_{\alpha} \int_{0}^{\pi} d \varphi \int_{0}^{2 \pi} w_{\alpha}^{v}\left(\hat{\lambda}_{\alpha}\right) h_{\alpha}(\varphi, \omega) \sin \varphi d \omega$,

where $N_{\alpha}$ is the total number of the $\alpha$-th kind of chains. $h_{\alpha}(\varphi, \omega)$ is the initial orientation distribution function of the $\alpha$-th kind of chains in the spherical coordinates $(r, \varphi, \omega)$, and satisfies the normalized condition $\int_{0}^{\pi} \int_{0}^{2 \pi} h_{\alpha}(\varphi, \omega) \sin \varphi d \varphi d \omega=1$. For isotropic viscoelastic material, the initial orientation distribution function is given by $h_{\alpha}=1 / 4 \pi$. Furthermore, we assume that the material is compressible and the total strain energy can be expressed by

$W=W(g)+\sum_{\alpha=1}^{M} W_{\alpha}^{v}\left(\hat{\lambda}_{\alpha}\right)$ 
where $g=\operatorname{det} \Lambda$ is the third invariant of the right stretch tensor $\Lambda$, and $W(g)$ is the strain energy of dilatation deformation. In the following, only two kinds of chains in the molecular network are considered, one with viscous dissipation, $W_{1}^{v}(\hat{\lambda})$, describing the strain rate sensitivity of polymeric material and another without viscous dissipation, $W_{0}(\lambda)$, describing the mechanical behavior at quasi-static loading. The specific forms of strain energy $W(g), W_{0}(\lambda)$ and $W_{1}^{v}(\hat{\lambda})$ are assumed to be

$W(g)=\frac{\lambda}{2}(\ln g)^{2}$,

$W_{0}(\lambda)=\frac{3 \mu \varepsilon_{0}^{2}}{m+1}\left\{\left[1+\frac{\varepsilon_{e f f}^{2}}{\varepsilon_{0}^{2}}\right]^{\frac{m+1}{2}}-1\right\}$,

$W_{1}^{v}(\hat{\lambda})=\frac{3 \overline{\mu \varepsilon}_{0}^{2}}{m^{\prime}+1}\left\{\left[1+\frac{\bar{\varepsilon}_{e f f}^{2}}{\bar{\varepsilon}_{0}^{2}}\right]^{\frac{m^{\prime}+1}{2}}-1\right\}$,

where $\lambda, \mu, \bar{\mu}, \varepsilon_{0}, \bar{\varepsilon}_{0}, m$ and $m^{\prime}\left(m, m^{\prime} \leq 1\right)$ are material parameters, $\varepsilon_{e f f}^{2}=(2 / 3)(\Lambda-\boldsymbol{I}):(\Lambda-\boldsymbol{I})$ and $\bar{\varepsilon}_{\text {eff }}^{2}=(2 / 3)(\Lambda-\boldsymbol{\xi}-\boldsymbol{I}):(\Lambda-\boldsymbol{\xi}-\boldsymbol{I})$. In view of the following relations:

$\frac{\partial g}{\partial \boldsymbol{E}}=g \boldsymbol{\Lambda}^{-1}$

$\frac{\partial \varepsilon_{e f f}}{\partial \boldsymbol{E}}=\frac{2}{3} \frac{\boldsymbol{E}}{\varepsilon_{e f f}}$,

the engineering stress $\boldsymbol{T}$, which is conjugate to Biot's strain $\boldsymbol{E}=\Lambda-\boldsymbol{I}$, may be expressed as

$\boldsymbol{T}=\frac{\partial W}{\partial \boldsymbol{E}}=\lambda(\ln g) \Lambda^{-1}+2 \mu\left[1+\frac{\varepsilon_{e f f}^{2}}{\varepsilon_{0}^{2}}\right]^{\frac{m-1}{2}}(\Lambda-\boldsymbol{I})+2 \bar{\mu}\left[1+\frac{\bar{\varepsilon}_{e f f}^{2}}{\bar{\varepsilon}_{0}^{2}}\right]^{\frac{m^{\prime}-1}{2}}(\Lambda-\boldsymbol{\xi}-\boldsymbol{I})$.

The generalized stress $\boldsymbol{Q}$, conjugate to the internal variable tensor $\boldsymbol{\xi}$, is similarly given by

$\boldsymbol{Q}=-\frac{\partial W_{1}^{v}}{\partial \xi}=2 \bar{\mu}\left[1+\frac{\bar{\varepsilon}_{e f f}^{2}}{\bar{\varepsilon}_{0}^{2}}\right]^{\frac{m^{\prime}-1}{2}}(\Lambda-\boldsymbol{\xi}-\boldsymbol{I})$.

The evolutionary equation of $\xi$ is usually assumed to obey the Onsager reciprocal relation

$\eta \dot{\boldsymbol{\xi}}=\boldsymbol{Q}$,

where $\eta$ is the viscous coefficient of the material. However, the Maxwell model with a nonlinear spring in series with a linear dashpot could not describe flexibly the rate sensitivities of the material in a wide range of strain rates. In the present model, a nonlinear evolution equation of $\xi$ is suggested as follows:

$\dot{\xi}=\frac{1}{\eta}\left(1+\frac{q_{e f f}^{2}}{q_{0}^{2}}\right)^{\frac{n-1}{2}} \boldsymbol{Q}$,

$\left.\xi\right|_{t=0}=0$,

where $\eta, q_{0}$ and $n(\geq 1)$ are material parameters and $q_{e f f}^{2}=(3 / 2) \boldsymbol{Q}: \boldsymbol{Q}$. 


\section{Comparison of predicted results with experimental data}

There are ten material parameters in the present constitutive model to describe the nonlinear viscoelastic response of polymeric material, i.e., Lamé's constants $\lambda, \mu$ and $\bar{\mu}$, the viscous coefficient $\eta$, the reference strain $\varepsilon_{0}$ and $\bar{\varepsilon}_{0}$, reference generalized stress $q_{0}$, nonlinear index $m$ and $m^{\prime}$ of springs and nonlinear index $n$ of the dashpot, respectively. It is expected that the mechanical response at various strain rates as well as quasi-static loading could be described by the present constitutive model. In order to verify the validity of the present model, uniaxial tension tests for HDPE are carried out on Instron 8510 with the geometry of the tensile specimens referred to ASTMD 638 type M. HDPE is in glass transition under the condition of room temperature. The influence of viscosity on the mechanical properties is rather obvious at the test temperature $18 \pm 2{ }^{\circ} \mathrm{C}$. Three strain rates are performed in the tensile experiments, i.e., $\dot{\lambda}_{1}=1 / 60(1 / s), \dot{\lambda}_{1}=1 / 150(1 / s)$, and $\dot{\lambda}_{1}=1 / 600(1 / s)$, respectively. The experimental data indicate that the higher loading rate may lead to the increase of rigidity and strength of the material. The present model is used to fit the experimental data. The least-square procedure based on a modified Levenberg-Marquardt algorithm is performed to obtain the model parameters, i.e., $\lambda=0.54(\mathrm{GPa}), \mu=0.36(\mathrm{GPa}), \bar{\mu}=0.25(\mathrm{GPa}), m=0.15, m^{\prime}=0.1, \varepsilon_{0}=$ $0.025, \bar{\varepsilon}_{0}=0.03, \eta=0.275(\mathrm{GPa} \cdot \mathrm{s}), n=7.0, q_{0}=15.0(\mathrm{MPa})$. Curves of the model prediction and the experimental data for three tensile strain rates are shown in Fig. 1. It is shown that the model prediction is in good agreement with the experimental data.

\section{Discussion of the present constitutive model}

It is assumed in Eq. (7) that the stress-strain behavior of polymeric solids can be decomposed into a quasi-static nonlinear elastic response and a rate-dependent response. The quasi-static nonlinear elastic response is described by a nonlinear spring, and the rate-dependent response by a generalized Maxwell model with a nonlinear spring in series with a nonlinear dashpot. The present constitutive model can be clearly illustrated with Fig. 2 . When the nonlinear index $m^{\prime}=n=1$, the generalized Maxwell model reduces to the standard Maxwell model with linear spring and dashpot and single relaxation time $\tau=\eta / \bar{\mu}$. However, the relaxation time $\tau$ in the present generalized Maxwell model is not a constant but dependent upon the deformation

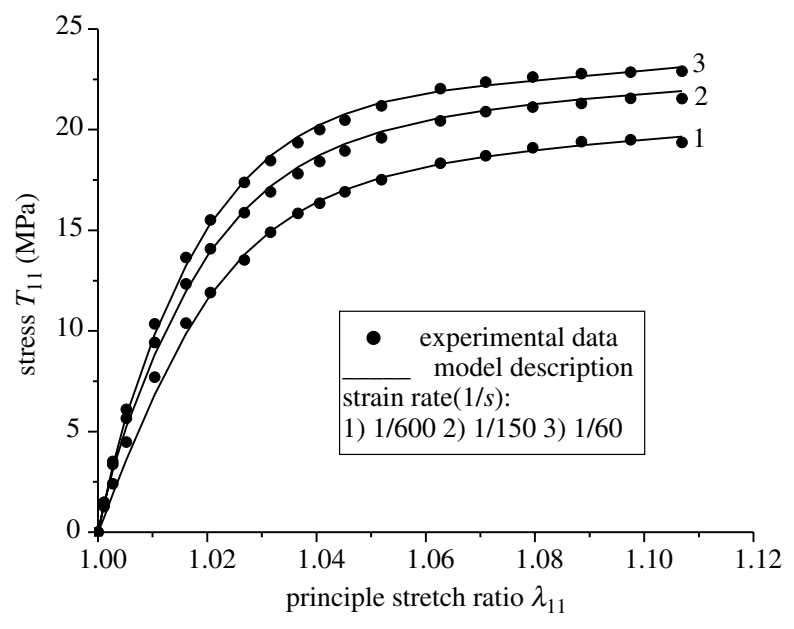

Fig. 1. The comparison between the model descriptions and the experimental data 


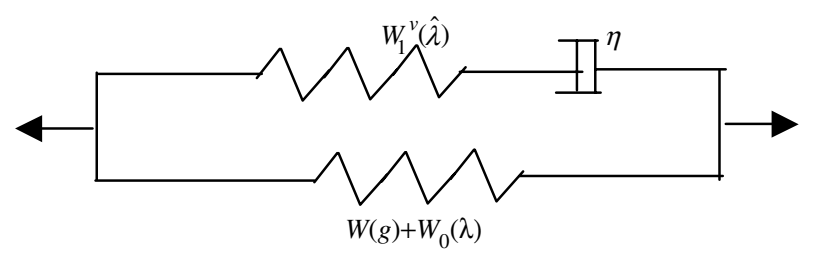

Fig. 2. The schematic interpretation of the present constitutive model---- one generalized Maxwell model in parallel with one nonlinear spring

process since both the tangent modulus of the nonlinear spring and the apparent viscosity coefficient of the nonlinear dashpot change in the deformation process. The relaxation time is an important parameter in a viscoelastic model, which affects not only the response history but also the range of rate-sensitivity. The objective of the present discussion is to understand how the relaxation time affects the material response and why the present constitutive model can describe the material response more flexibly. It is recognized that the relaxation time is related to the mode of micro-structure motion. Because there are many motion elements with different sizes in molecular networks, more than one relaxation time constant is usually needed to describe the mechanical behavior in the full range of strain rates. It results in the generalized Maxwell model, the combination of many Maxwell elements with relaxation time spectrum. However, too many material parameters in a constitutive model are inconvenient for practical application. An appropriate compromise between minimizing the material parameters and simulating closely the material response is inevitable. Yang et al. [9] used a Maxwell model with single relaxation time constant to describe the large deformation response of an incompressible rubber material under high strain rates, and found that the range of rate-sensitivity captured by their model was $10^{-2} \sim 10^{0}$, i.e. two orders of magnitude, for non-dimensional strain rates $\dot{\lambda}=\tau \dot{\lambda}$. Wang et al. [10] proposed a constitutive model with two relaxation time constants, called "ZWT" model, whereby one relaxation time was used to describe the mechanical behavior at high strain rates $\left(10^{2} \sim 10^{3} \mathrm{~s}^{-1}\right)$ and another one to describe the mechanical behavior at low strain rates $\left(10^{-4} \sim 10^{-1} \mathrm{~S}^{-1}\right)$. The range of rate-sensitivity captured by their model was examined and it was pointed out that a relaxation time has an effective influence domain of about 4.5 orders of magnitude. It is noted that the range of rate-sensitivity is different in the two models mentioned above. The difference results from the introduction of a nonlinear spring in the model of Yang et al. instead of the linear spring in the "ZWT" model. The observation reveals the fact that the generalized Maxwell model with nonlinear spring can only describe ratesensitive behavior in a less narrow range than a standard Maxwell model with linear spring and dashpot. In order to investigate in detail the effects of a nonlinear spring and dashpot on the range of rate-sensitivity, the response of eleven strain rates by a standard Maxwell model and generalized Maxwell model with nonlinear spring or dashpot is simulated and the numerical results are shown in Fig. 3. The stress and the strain rates in the figures are nondimensionalized by the initial elastic modulus of the spring and the initial relaxation time, respectively, but the same symbols are used, namely, $T / \bar{\mu} \Rightarrow T$ and $\dot{\lambda} \cdot \tau \Rightarrow \dot{\lambda}$. It can be seen clearly from Fig. $3 \mathrm{~b}$ that the range of rate-sensitivity of a generalized Maxwell model with a nonlinear spring shrinks significantly, and further observation shows that the range of rate-sensitivity shifts toward low strain rates. It may be explained by the change of relaxation time in the deformation process. Considering that the viscosity coefficient of a dashpot does not change but the tangent modulus of a nonlinear spring decreases gradually during deformation, it is found that the relaxation time increases gradually during deformation, and this results in the shift of the range of ratesensitivity toward the low strain rates. The numerical simulation results by another generalized Maxwell model with linear spring but nonlinear dashpot are shown in Fig. 3c. Surprisingly, the range of rate-sensitivity extends distinctively as compared to the standard Maxwell model. This 

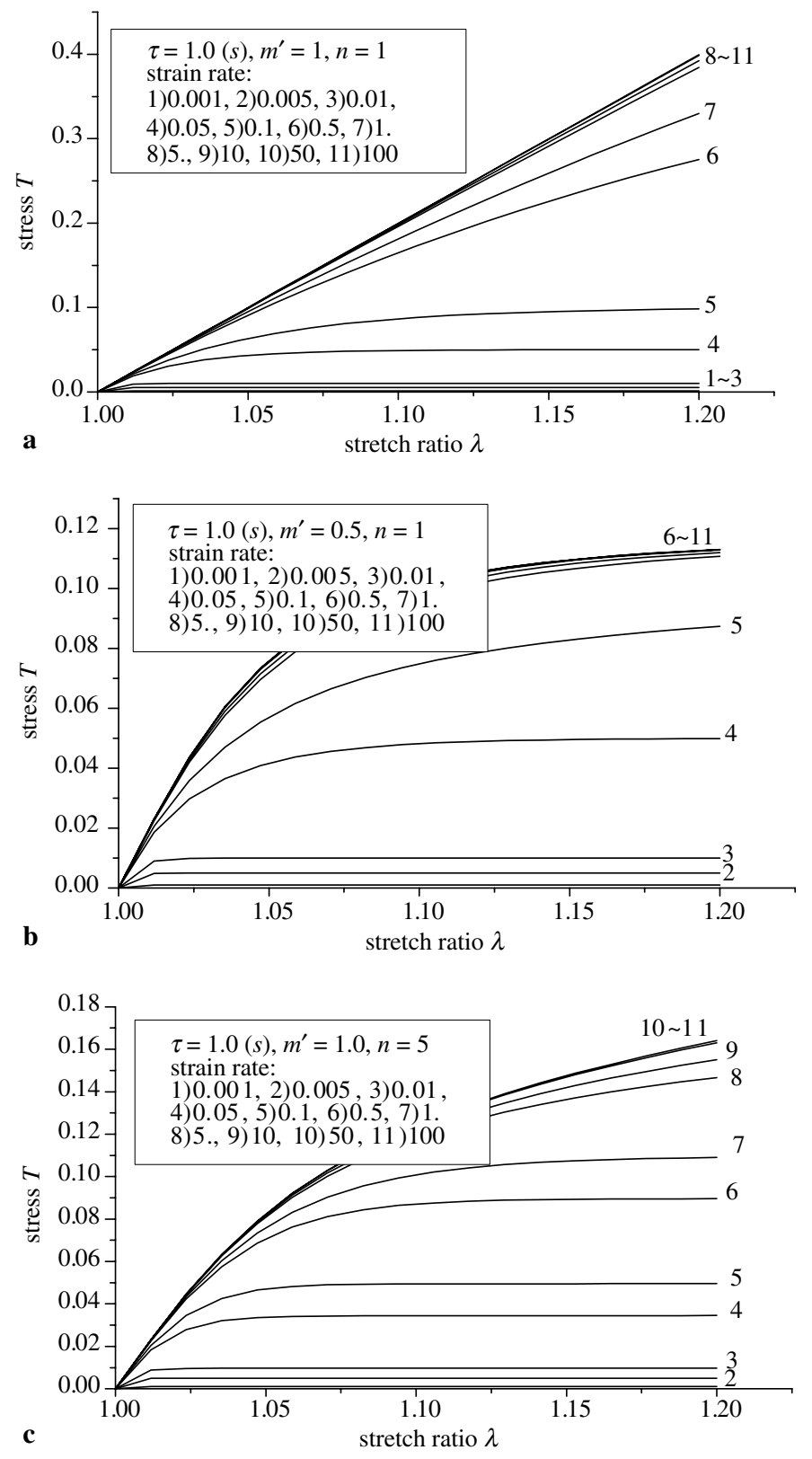

Fig. 3. The range of rate-sensitivity of three Maxwell models; (a) linear spring and dashpot, (b) nonlinear spring and linear dashpot, (c) nonlinear dashpot and linear spring

phenomenon can similarly be explained by the change of relaxation time during deformation. The change of the apparent viscosity coefficient of a nonlinear dashpot makes the relaxation time decreasing gradually during deformation, and therefore makes the range of rate-sensitivity extended significantly. These observations give an implication that the generalized Maxwell model with nonlinear spring describes rate sensitivity in a narrow range as compared to the standard Maxwell model. However, the generalized Maxwell model with nonlinear dashpot can describe the rate sensitivity in a wider range.

The present constitutive model includes a generalized Maxwell model with nonlinear spring and nonlinear dashpot. Because of the continuous change of the tangent modulus of the nonlinear spring and the apparent viscosity coefficient of the nonlinear dashpot during 

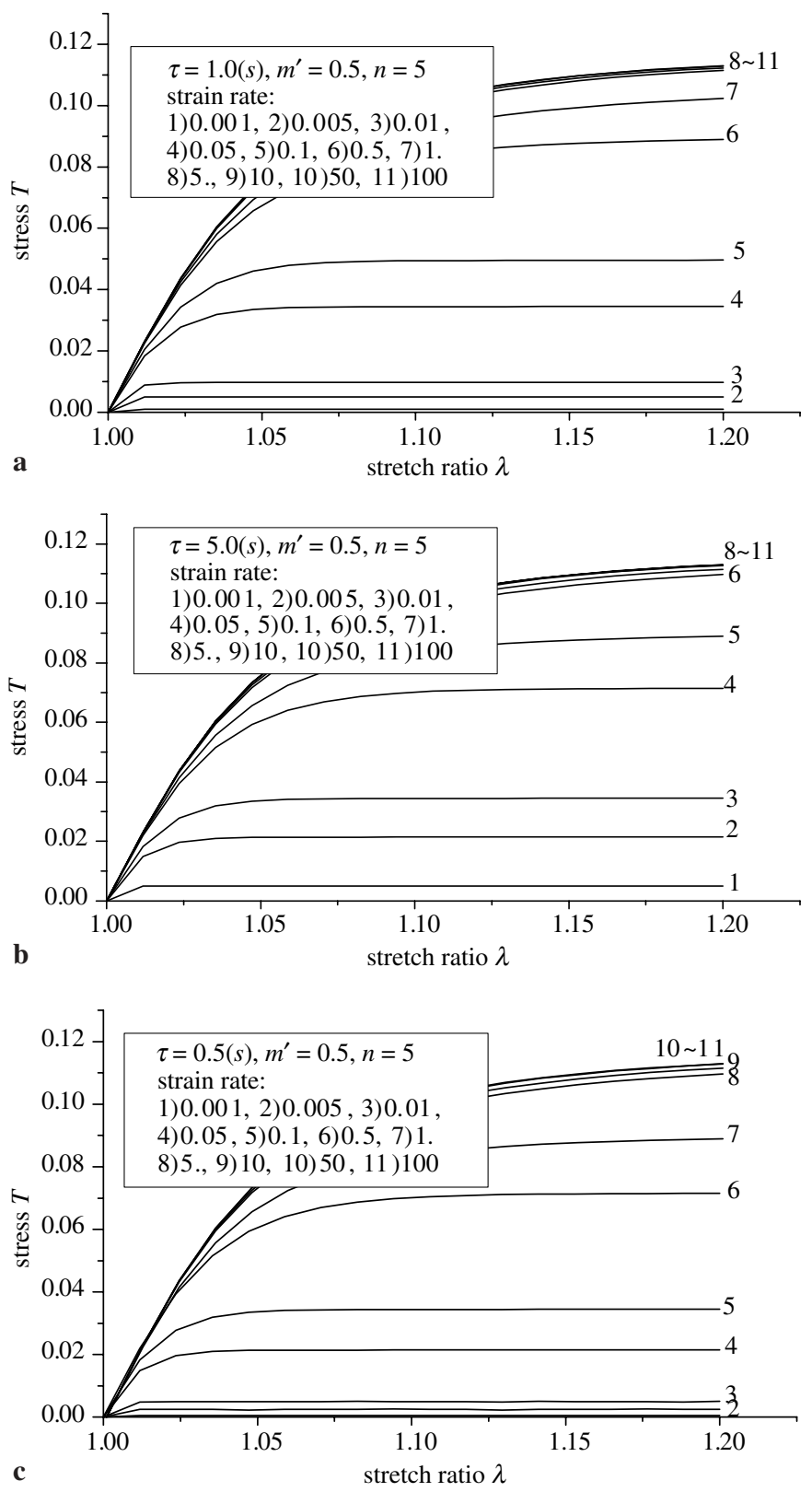

Fig. 4. The effects of initial relaxation time on the range of rate-sensitivity, (a) medium relaxation time, (b) longer relaxation time, $(\mathbf{c})$ shorter relaxation time

deformation, the effect of the initial relaxation time on rate sensitivity becomes another concern. Three initial relaxation times, with the other material parameters fixed, are simulated numerically and illustrated in Fig. 4. It is shown clearly that the range of rate-sensitivity shifts toward low strain rates when the initial relaxation time increases (compare (a) with (b)) and toward high strain rates when the initial relaxation time decreases (compare (a) with (c)).

The last concerns are the effect of the nonlinear index on rate-sensitivity. Figures $3 \mathrm{c}, 4 \mathrm{a}$ and 5 a correspond to three nonlinear indices of the spring, i.e., $m^{\prime}=1.0,0.5,0.3$, with other material parameters identical. It is observed that decreasing the nonlinear index of the spring results in the reduction of the range of rate-sensitivity. Figures $3 b, 5 b$ and $4 a$ correspond to three nonlinear indices of the dashpot, i.e., $n=1,3,5$, with the other material parameters 

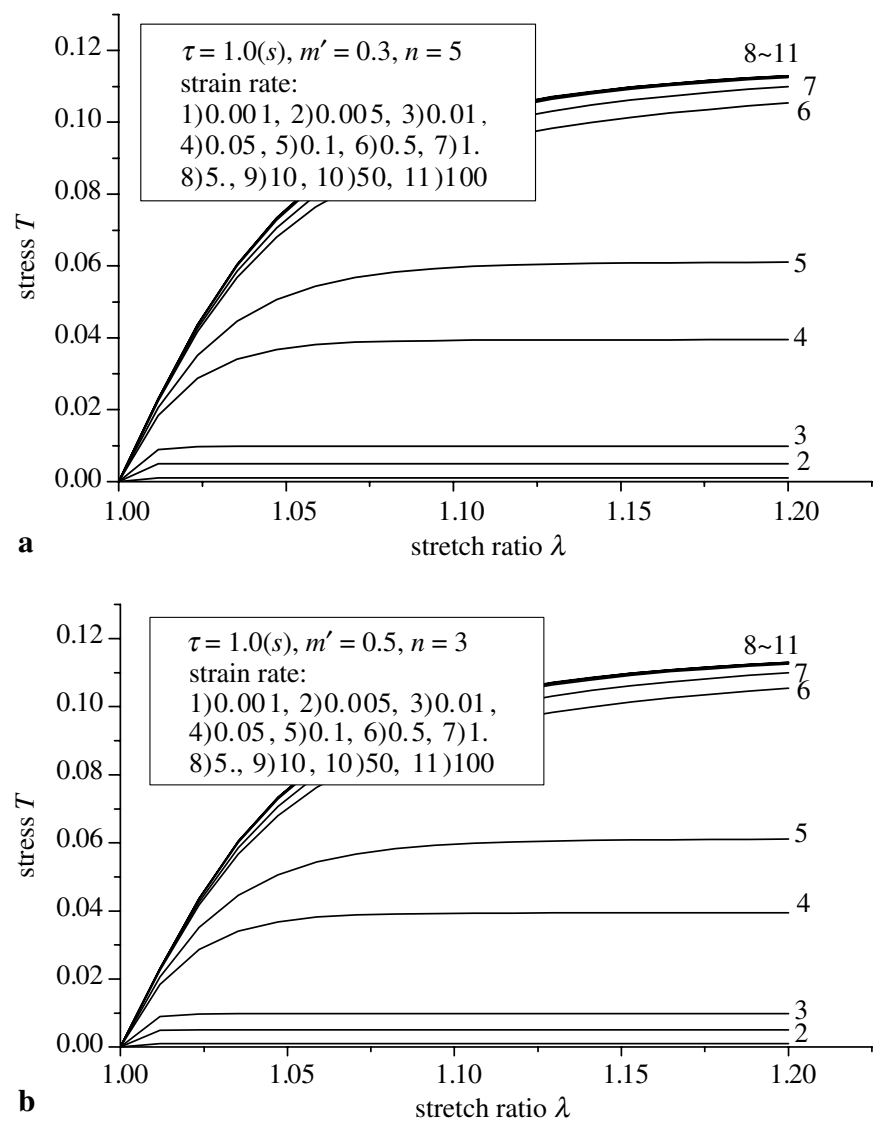

Fig. 5. The effects of the nonlinear index on the range of ratesensitivity, (a) small index for spring and large index for dashpot, (b) medium index for spring and dashpot

identical. It is observed that increasing the nonlinear index of the dashpot results in the extension of the range of rate-sensitivity.

\section{Concluding remarks}

By introducing a set of second rank tensorial internal variables corresponding to Biot's strain, the network theory in rubber elasticity is extended to incorporate the viscous effects of polymeric materials. In order to describe properly the rate-sensitivity of the material, a nonlinear evolution equation for the internal variables is proposed. The physical explanation of the present constitutive model is the combination of a nonlinear spring which describes the quasistatic response and a generalized Maxwell model with nonlinear spring and nonlinear dashpot which describes the rate-sensitivity response of materials. Uniaxial tension tests of HDPE are carried out for three strain rates. It is found that the prediction of the present model is in good agreement with the experimental data. Hence, the present model can describe adequately the viscoelastic response of polymeric materials at different strain rates. The range of rate-sensitivity is studied in detail for the present model with nonlinear spring and nonlinear dashpot. The numerical simulation demonstrates that the introduction of a nonlinear spring results in the reduction of the range of rate-sensitivity and the introduction of a nonlinear dashpot results in the extension of the range of rate-sensitivity. The standard Maxwell model with linear spring and dashpot keeps the relaxation time, $\tau=\eta / \bar{\mu}$, constant. However, in the present generalized 
Maxwell model with nonlinear spring and dashpot, changes of the tangent modulus of the nonlinear spring and the apparent viscosity coefficient of the nonlinear dashpot in the deformation process result in the change of the relaxation time $\tau$, which forms a continuous relaxation time spectrum. The investigation of the effect of initial relaxation time demonstrates that a longer initial relaxation time makes the range of rate-sensitivity shifting toward low strain rates and a shorter initial relaxation time makes the range of rate-sensitivity shifting toward high strain rates. In addition, the nonlinear indices of spring and dashpot affect significantly the range of rate sensitivity. All these observations indicate that the range of rate-sensitivity can be flexibly changed by adjusting the initial relaxation time and the nonlinear index of spring and dashpot when the evolutionary law of the internal variables is given. Hence the present constitutive model is more flexible to describe the strain rate sensitivity of polymeric material in a wide range of strain rates.

\section{Acknowledgement}

The authors thank Prof. Z. P. Huang for instructive suggestions and discussions and reading the early version of the manuscript. This work was supported by the National Natural Science Foundation of China (No.10272003, No.10032010, 10172074) and Natural Science Foundation of Yangzhou University.

\section{References}

[1] Christensen, R. M.: A nonlinear theory of viscoelasticity for application of elastomers. J. Appl. Mech. 47, 762-768 (1980).

[2] Gurtin, M. E., Hrusa, W. J.: On the thermodynamics of viscoelastic materials of single-integral type. Quart. Appl. Math. 49, 67-85 (1991).

[3] Drozdov, A. D.: Mechanics of viscoelastic solids. New York: Wiley 1998.

[4] Chen, X. H., Tong, P., Wang, R.: Nonequilibrium statistical thermodynamic theory for viscoelasticity of polymers. J. Mech. Phys. Solids. 46, 139-152 (1998).

[5] Biot, M. A.: Theory of stress-strain relations in anisotropic viscoelasticity and relaxation phenomena. J. Appl. Phys. 25, 1385-1391 (1954).

[6] Coleman, B. D., Gurtin, M. E.: Thermodynamics with internal state variables. J. Chem. Phys. 47, 597-613 (1967).

[7] Holzapfel, G. A., Simo, J. C.: A new viscoelastic constitutive model for continuous media at finite thermomechanical changes. Int. J. Solids. Struct. 33, 3019-3034 (1996).

[8] Huang, Z. P., Chen, J. K., Wang W. B.: An internal variable theory of thermo-viscoelastic constitutive relations at finite strain. Science in China 43, 545-551(2000).

[9] Yang, L. M., Shim, V. P. W., Lim, C. T.: A visco-hyperelastic approach to modelling the constitutive behaviour of rubber. Int. J. Impact Engng. 24, 545-560 (2000).

[10] Wang, L.L., Yang, L. M.: A class of nonlinear viscoelastic constitutive relations of solid polymeric material. In: The progress in impact dynamics. (Wang, L. L., Yu, T. X., Li, Y. C., eds.), pp. 88-116. Hefei: Chinese Sciences and Technology University Press 1992.

Authors' addresses: P. J. Wei, Laboratory of Nonlinear Mechanics of Continuous Media (LNM), Institute of Mechanics, Chinese Academy of Sciences and Department of Mechanics and Engineering Sciences, Peking University, Beijing, P. R. China (E-mail: weipj@pku.edu.cn); J. K. Chen, Department of Hydraulic and Hydroelastric Engineering, Yangzhou University, Yangzhou, P. R. China

Verleger: Springer-Verlag KG, Sachsenplatz 4-6, A-1201 Wien. - Herausgeber: Prof. Dipl.-Ing. Dr. Hans Troger und Prof. Dipl.-Ing. Dr. Franz Ziegler, beide Technische Universität, Wiedner Hauptstraße 8-10, A-1040 Wien. - Redaktion: Wiedner Hauptstraße 8-10, A-1040 Wien. Hersteller: Scientific Publishing Services (P) Ltd., Madras, India. - Offsetdruck: Manz Crossmedia, A-1051 Wien. - Verlagsort: Wien. Herstellungsort: Wien. - Printed in Austria. 